\title{
Fruit juice of silverberry (Elaeagnus) and bayberry (Myrica) may help in combating against kidney dysfunctions
}

Pallab Kar ${ }^{1}$, Arnab Kumar Chakraborty', Somit Dutta ${ }^{1,2}$, Malay Bhattacharya ${ }^{3}$, Tapas Kumar Chaudhuri ${ }^{2,4}$ and Arnab Sen ${ }^{1 *}$

\begin{abstract}
Background: Elaeagnus pyriformis, Myrica nagi and Myrica esculenta are prominent actinorhizal plants of NorthEast India which produce juicy edible fruits. Native people of this region consume these fruits for nutritional enhancement. Apart from having immense nutritional value, fruits of these plants have been utilized in traditional medicine for providing protection against various ailments. In our previous study on antioxidant activity and phytochemical fingerprints using GC-MS method; many medicinally potent compounds in fresh fruit juice of the three plants were revealed. We reported the presence of several compounds used to cure renal ailments. In the present study we reported the efficacy of these juices to alleviate nephrotoxicity in rat model. We intend to understand to what extent these fruits could be beneficial. Within the same scope we tried to evaluate whether the bioactive compounds present in the fruits could be responsible for reducing nephrotoxicity by in-silico molecular docking approaches.
\end{abstract}

Methods: In this study, three fruit juices namely Elaeagnus pyriformis, Myrica nagi and M. esculenta were evaluated on gentamicin induced nephrotoxicity in rat model. Nephrotoxicity was induced in wistar albino rats by intraperitoneal administration of gentamicin ( $100 \mathrm{mg} / \mathrm{kg} /$ day body weight) for 7 days. The study included a control group which received oral saline only, negative control group received gentamicin, positive control group received gentamicin and cystone, low dose group received gentamicin along with juice $(200 \mathrm{mg} / \mathrm{kg} /$ day) and high dose group received gentamicin along with juice ( $400 \mathrm{mg} / \mathrm{kg} /$ day). Various enzymatic and biochemical parameters were assessed to study the protective effect of fruit juices in kidney disorders.

Results: Among the three fruits M. esculenta showed promising result in all in-vivo enzymatic assays (Superoxide dismutase, Glutathione reductase, Lipid peroxidation and catalase). Nevertheless, all the three fruit juice showed high significance value with encouraging range of percentage of significance. The histological slides and in-silico study confirmed that these fruits are promising in alleviating nephrotoxicity in rat model.

Conclusion: We may therefore conclude that fruit juices of silverberry (Elaeagnus) and bayberry (Myrica) may be used for formulating herbal medicine as a potent scavenger of free radicals to prevent the toxic effects of gentamicin in kidney disorders.

Keywords: Actinorhizal plants, Gentamicin, Catalase, Histology, Molecular docking

\footnotetext{
* Correspondence: senarnab_nbu@hotmail.com

${ }^{1}$ Department of Botany, University of North Bengal, Siliguri, West Bengal

734013, India

Full list of author information is available at the end of the article
} 


\section{Introduction}

Kidneys are a pair of organs in mammals and other nonmammalian vertebrates essential for the functions including osmoregulation, excretion of end products like urea and creatinine, synthesis of hormones such as rennin and erythropoietin and for the metabolism of vitamin $\mathrm{D}$. To help them perform all these activities smoothly kidneys have the richest blood supply per unit weight of tissue in the body [1]. Blood supply to the kidneys is by the renal arteries and venous drainage is by the renal veins. The renal vessels are of such high calibre that the vascular bed receives a large volume of toxinborne blood (about $20-25 \%$ of resting cardiac volume) $[2,3]$. The cells of the kidney (especially, the cells of the proximal convoluted tubules) thus become prone to injury from natural or synthetic agents such as antimicrobials, immune suppressants, chemotherapeutic agents and analgesics [4]. For instance, Gentamicin, an aminoglycoside antibiotic which is extensively used against the treatment of severe Gram negative bacterial infections, has a nephrotoxic complication associated with it [5]. Gentamicin related nephrotoxicity mainly induces initial oxidative stress in kindey that is further compounded with reduction in natural antioxidant defence mechanisms $[5,6]$. Apart from allopathic treatment, there are herbal formulations which are effective against kidney ailments such as Clerodendrum myricoides and Aralia leschenaultii [7]. Native people of north-east India also use actinorhizal fruits (both pulp and juice) against various ailments.

Actinorhizal plants are taxonomically diverse group of dicotyledonous angiosperms. They are mostly woody trees or shrubs, except for the genus Datisca which is herbaceous in nature [8]. Elaeagnus pyriformis, Myrica nagi and Myrica esculenta are three actinorhizal edible fruitbearing plants mainly found in north-eastern India which is de-facto a biodiversity hub (Fig. 1). Native people of this region consume these fruits for nutritional enhancement. Apart from having immense nutritional value, fruits of these plants have been utilized in traditional medicine for providing protection against various ailments together with kidney diseases. Elaeagnus (Silverberry or Oleaster) is a deciduous, shrub belonging to the family Elaeagnaceae. In India, only four species of Elaeagnus are recorded namely E. pyriformis, E. angustifolia, E. latifolia and E. umbellata [9]. Myrica (Bayberry or Box myrtle) belonging to the family Myricaceae varies between shrubs to trees and is mostly evergreen, dioecious with height ranging from 3 to $10 \mathrm{~m}$. We previously reported comparative antioxidant profiling [10] and phytochemical fingerprints [11] of Elaeagnus and Myrica. Kar et al. [11] also reported the presence of several compounds used to cure renal ailments. Therefore, the aim of the present study was to find out the efficacy of the actinorhizal fruit juices (AFJs) in curing nephrotoxicity using rat model. We intend to understand to what extent these fruits could be beneficial to the people. Within the same scope we tried to evaluate whether the bioactive compounds present in these fruits could be responsible for reducing nephrotoxicity by insilico molecular docking approaches.

\section{Materials and methods}

Plant materials and extraction

The fruits of Elaeagnus pyriformis (EP), Myrica nagi (MN) and M. esculenta (ME) were collected during March 2017, from the local market of Shillong, Meghalaya, India. The fruits were identified by plant taxonomist of Department of Botany, University of North Bengal. The voucher specimens (Accn. \# 9660, 9659 and 9658 for E. pyriformis, M. nagi and $M$. esculenta respectively) were deposited at the Herbarium of the same department. The fresh fruits (500 g) were washed twice with double distilled water and surface-dried at room temperature. The fruits were mechanically squeezed into juice $(100 \mathrm{ml})$ after separation of the seeds. The juice obtained was stored at $4{ }^{\circ} \mathrm{C}$ for further use.

\section{Ethical statement}

The fruits of Elaeagnus pyriformis, Myrica nagi and $M$. esculenta used in the present study did not belong to
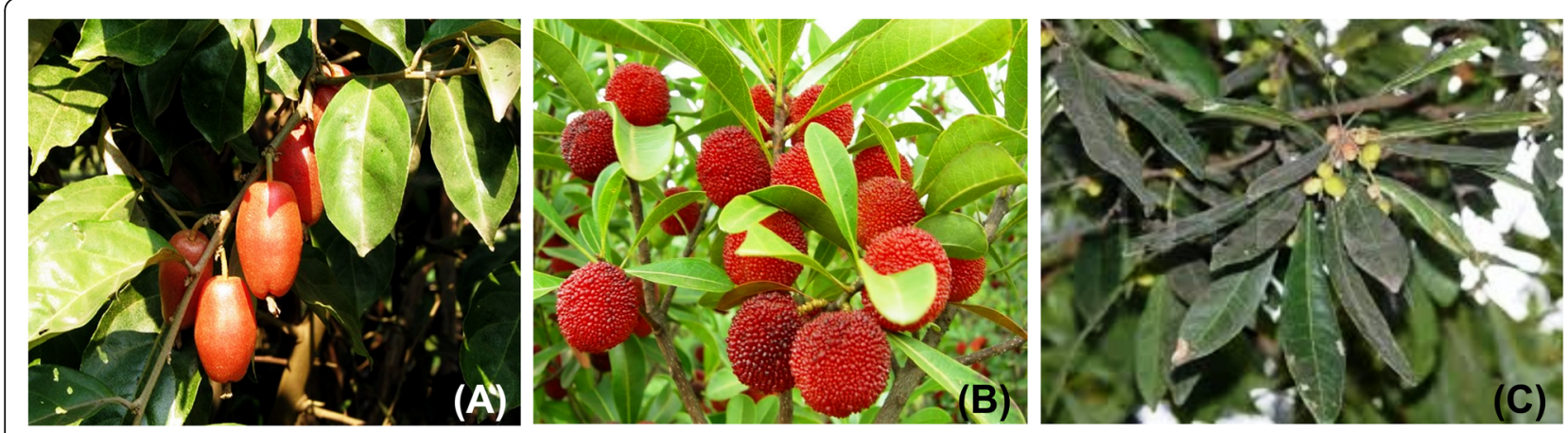

Fig. 1 Plants bearing fruits of a Elaeagnus pyriformis, b Myrica nagi, c Myrica esculenta 
any endangered or protected species. The collection area of the fruit samples are not under a National Park/Reserve Forest/Govt. protected area.

\section{Animals and care}

Healthy male Wistar albino rats (10-12 weeks of age and $78 \pm 4$ g body weight) were used for all the experiments. Rats ( 4 male/group, $n=4)$ were kept in polypropylene side cage bins (Tarson, India), with rice husk as bedding material. The experimental rats were maintained in the animal house of the Department of Zoology, University of North Bengal with sufficient food and water ad-libitum under a constant $12 \mathrm{~h}$ dark/light cycle at an environmental temperature of $25 \pm 2{ }^{\circ} \mathrm{C}$ and humidity $55 \pm 5 \%$.

\section{Acute toxicity study}

OECD guidelines (test 423: Acute oral toxicity-Acute toxic class method; 2002) were followed to study the acute toxicity of the three fruit juices [12]. All the animals (12 male Wistar albino rats) were kept on fasting overnight prior to the experiment. The fruit juices were administered orally in an increasing dose of 250,500, 1000 and $2000 \mathrm{mg} / \mathrm{kg}$ body weight (BW). The concentrations are determined as per OECD guideline. After feeding of various doses of formulation, all the rats were carefully observed for development of clinical or toxicological symptoms at $30 \mathrm{~min}$ and then at 2, 4, 8, 24 and $48 \mathrm{~h}$.

\section{Experimental design and drug administration External drugs}

We used Cystone ${ }^{\circ}$ as standard drug and positive control for our study. Cystone is known for its Ayurvedic formulation for the treatment of kidney ailments, manufactured by Himalayan Herbal health care. For details about Cystone please refer http://www.himalayawellness.com/ research/cystone.htm

Wistar albino rats (36 male) were randomly divided into nine groups $(n=4)$ and following treatments were done once per day for 7 consecutive days to induce experimental nephrotoxicity in rats:

Group I: Control- received normal saline for 7 days;

Group II: Negative control group (Gentamicin treated group)- injected with gentamicin $(100 \mathrm{mg} / \mathrm{kg} /$ day $\mathrm{BW})$ intraperitonially for 7 days;

Group III: Positive control group (Cystone treated group) administrated gentamicin $(100 \mathrm{mg} / \mathrm{kg} /$ day $\mathrm{BW})$ intraperitonially along with cystone $(400 \mathrm{mg} / \mathrm{kg} /$ day BW; dissolved in Mili-Q water) orally for 7 days;

Group IV, VI, VIII: Low dose group (EP, MN, ME)- administrated gentamicin $(100 \mathrm{mg} / \mathrm{kg} /$ day $\mathrm{BW})$ intraperitonially along with juice $(200 \mathrm{mg} / \mathrm{kg} /$ day BW; dissolved in Mili-Q water) orally for 7 days;
Group V, VII, IX: High dose group (EP, MN, ME)- administrated gentamicin $(100 \mathrm{mg} / \mathrm{kg} /$ day $\mathrm{BW})$ intraperitonially along with juice $(400 \mathrm{mg} / \mathrm{kg} /$ day BW; dissolved in Mili-Q water) orally for 7 days.

\section{Preparation of kidney tissue samples}

On 8th day i.e. $24 \mathrm{~h}$ after the last dose, under proper anesthesia (mild 2\% ether) blood was collected in an EDTA-containing tube by puncturing the heart from Wistar albino rats and then all the animals were sacrificed by cervical dislocation. Blood was allowed to clot for $60 \mathrm{~min}$ at room temperature $\left(25^{\circ} \mathrm{C}\right)$ and then serum was collected by low speed centrifugation at $120 \mathrm{x} g$ for $5 \mathrm{~min}$. The straw colored serum was used to study the biochemical parameters. After blood collection, both kidneys of each group was removed and washed with phosphate buffer saline to remove blood followed by homogenization in phosphate buffer $(50 \mathrm{mM}$; pH 7.4) and potassium chloride $(\mathrm{KCl} ; 120 \mathrm{mM})$. The homogenate was centrifuged at $1000 \mathrm{x} \mathrm{g}$ for $10 \mathrm{~min}$ at $4{ }^{\circ} \mathrm{C}$. The supernatant was collected after centrifugation and used for in-vivo enzymatic assays [13]. The remaining kidney was preserved in $10 \%$ formaldehyde solution for histological study.

\section{Serum biochemical assays}

Serum samples from each group were used to study kidney function tests like creatinine and urea levels using commercially available kits (Crest Biosystems, India).

\section{In-vivo kidney enzymatic assays}

Estimation of superoxide dismutase (SOD) For the estimation of superoxide dismutase, standard method was followed with minor modifications [14]. Reaction mixture was prepared using $1 \mathrm{ml}$ of $50 \mathrm{mM}$ sodium carbonate, $0.4 \mathrm{ml}$ of $25 \mu \mathrm{M}$ nitroblue tetrazolium and $0.2 \mathrm{ml}$ of $0.1 \mathrm{mM}$ freshly prepared hydroxylamine hydrochloride. Clear supernatant of kidney homogenate $(0.1 \mathrm{ml}, 1: 10 \mathrm{w} /$ v) was added to the reaction mixture. The changes in absorbance of the sample were recorded at $560 \mathrm{~nm}$.

Estimation of reduced glutathione (GSH) Reduced glutathione activity was measured according to the standard protocol [15] . An aliquot of $1 \mathrm{ml}$ kidney tissue supernatant was treated with 0.5 of Elman reagent (19.8 $\mathrm{mg}$ DTMB dissolved in $100 \mathrm{ml}$ of $0.1 \%$ sodium nitrate). After the treatment with Elman reagent, $3 \mathrm{ml}$ of phosphate buffer was added and the absorbance was measured at $412 \mathrm{~nm}$.

Measurement of lipid peroxidation (MDA) The MDA content was determined using TBARS assay kit (Cayman, USA) according to the manufacturer's instructions. In 
brief, $100 \mu \mathrm{l}$ of clear supernatant of kidney homogenate of different groups were mixed with $100 \mu \mathrm{l}$ sodium dodecyl sulphate (SDS) solution. The tubes were placed in boiling water bath after addition of $4 \mathrm{ml}$ colour reagent. The tubes were kept on ice for $10 \mathrm{~min}$ to stop the reaction after 60 min incubation. Then the solutions were centrifuged $(1600 \mathrm{~g})$ for $10 \mathrm{~min}$ at $4{ }^{\circ} \mathrm{C}$ and the absorbance of the supernatants was recorded at $340 \mathrm{~nm}$.

Estimation of catalase activity Catalase activity was assessed by the standard protocol of Luck [16] with some modifications, wherein degradation of substrate $\mathrm{H}_{2} \mathrm{O}_{2}$ by catalase in the kidney tissue samples was measured. In a spectrophotometric cuvette, $500 \mu \mathrm{l}$ of 0.34 $\mathrm{mM} \mathrm{H}_{2} \mathrm{O}_{2}, 2.5 \mathrm{ml} \mathrm{H}_{2} \mathrm{O}$ and $40 \mu \mathrm{l}$ supernatant were added and change in absorbance was noted at $240 \mathrm{~nm}$.

Histopathological examination Kidneys from the experimental rat were removed and fixed in $10 \%$ formaldehyde solution for overnight followed by dehydration. Dehydrated tissues were embedded in paraffin and cut into $4 \mu \mathrm{m}$ sections using microtome. Then kidney sections were dewaxed in xylene, rehydrated in a series of different grades of alcohol and then washed with distilled water for $5 \mathrm{~min}$. The kidney sections were stained with basic stain haematoxylin for $40 \mathrm{~s}$ and counterstained with acidic stain eosin for $20 \mathrm{~s}$ [17]. After proper staining the slides were observed (100X and 400X) using Nikon ECLIPS E200 microscope.

Molecular docking Prominent and biologically active compounds present in three fruits juices were detected through GC-MS analysis like 1,2-Longidione, 2-Butenedioic acid (E)-, monomethyl ester, 5-Hydroxymethylfurfural, Adrenalone, n-Hexadecanoic acid etc. [11]. These biologically active chemicals were selected to further identify the most effective ligand molecule from in-silico molecular docking analysis. The search for a probable receptor molecule for the same was literature based. The protein selected here was Polycystic Kidney Disease protein 2 (5T4D), which is primarily an ion-channel regulator involved in production of a protein named polycystin in kideny [18]. The protein is selected keeping in mind that the in-vivo experiments are supported by the in-silico analysis. Polycystic Kidney Disease protein is related to calcification of kidney [19]. The Xray crystallographic structure of the protein available in the Protein Data Bank (http://www.rcsb.org) was used. Molecular docking was conducted using AutoDockVina [20]. The receptor structure was defined as rigid, and the grid dimensions were 100 for all three X, Y, and Z axes. Gasteiger charges were assigned for all the compounds, and nonpolar hydrogen atoms were merged. The ligands were the phytocompounds detected by GC-MS analysis having reported biological activities. While preparing the ligand for docking all the torsions were allowed to rotate. The value for the exhaustiveness of the search was 8. All graphic manipulations and visualizations were performed using the AutoDock Tools and ligand docking with AutodockVina.

Statistical analysis For reproducibility, all data were prepared as the mean \pm SD of six measurements. Statistical analysis were performed by one-way analysis of variance (ANOVA) with Dunnett's test using KyPlot version 5.0 (32 bit) for windows. $P<0.05$ was considered as significant.

\section{Results}

\section{Acute toxicity study}

The three fruit juices were administered orally and no mortality was observed in the experimental animals at $2000 \mathrm{mg} / \mathrm{kg}$ dose. Therefore, $1 / 10$ th $(200 \mathrm{mg} / \mathrm{kg})$ and $1 /$ 5 th $(400 \mathrm{mg} / \mathrm{kg})$ of the maximum dose were considered safe for the in-vivo studies.

\section{Body weight changes}

Changes in rat's body weight after the treatment of gentamicin, cystone and three fruit juices (EP, MN and ME) have been shown in Table 1. In the treatment period, the body weight of control rat increased by around $4 \%$ while the gentamicin treated rat lost the weight by around $5 \%$. This shows that body weight loss takes place during kidney ailment. Cystone treated rat gained 2\% body wt. which is half the body wt. gained by the healthy rat during treatment period. Interestingly, all our fruit juices treated rat gained more body wt. than the Cystone treated rat. Low dose EP $(200 \mathrm{mg} / \mathrm{kg} /$ day BW) (3.65\%) and high dose ME (400 mg/kg/day BW) (3.63\%) attained highest weight gain.

\section{Serum biochemical assays}

The serum biochemical assay of present study has been tabulated in Table 2. In control group, creatinine and

Table 1 Effects of three actinorhizal fruits on the body weight of the treated rat

\begin{tabular}{lll}
\hline Group & Body weight (Percentile) & \% body weight change \\
\hline Control & $104.54 \pm 0.95$ & $4.54 \pm 0.95 \boldsymbol{\Delta}$ \\
Gentamicin & $94.81 \pm 0.69$ & $5.18 \pm 0.69 \boldsymbol{\nabla}$ \\
Cystone & $102.33 \pm 0.57$ & $2.33 \pm 0.57 \boldsymbol{\Delta}$ \\
EP $200 \mathrm{mg} / \mathrm{kg}$ & $103.65 \pm 0.06$ & $3.65 \pm 0.06 \boldsymbol{\Delta}$ \\
EP $400 \mathrm{mg} / \mathrm{kg}$ & $102.36 \pm 0.23$ & $2.36 \pm 0.23 \boldsymbol{\Delta}$ \\
MN $200 \mathrm{mg} / \mathrm{kg}$ & $102.41 \pm 0.19$ & $2.41 \pm 0.19 \boldsymbol{\Delta}$ \\
MN $400 \mathrm{mg} / \mathrm{kg}$ & $102.98 \pm 0.53$ & $2.98 \pm 0.53 \boldsymbol{\Delta}$ \\
ME $200 \mathrm{mg} / \mathrm{kg}$ & $102.93 \pm 0.83$ & $2.93 \pm 0.83 \boldsymbol{\Delta}$ \\
ME $400 \mathrm{mg} / \mathrm{kg}$ & $103.63 \pm 0.27$ & $3.63 \pm 0.27 \boldsymbol{\Delta}$ \\
\hline
\end{tabular}

Weight (mean \pm SD) in gram, $\boldsymbol{\Lambda}$ Increase weight; $\boldsymbol{\nabla}$ Decrease weight 
Table 2 Effects of three actinorhizal fruits on serum biochemical parameters of nine $(n=9)$ treated groups

\begin{tabular}{|c|c|c|c|c|c|c|}
\hline \multirow[t]{2}{*}{ Group } & \multicolumn{3}{|c|}{ Creatinine (mg/dl) } & \multicolumn{3}{|l|}{ Urea $(\mathrm{mg} / \mathrm{dl})$} \\
\hline & Value & Inc./Dec. from control & Inc./Dec. from Gentamicin & Value & Inc./Dec. from control & Inc./Dec. from Gentamicin \\
\hline Control & $0.65 \pm 0.02$ & - & - & $44.42 \pm 2.19$ & - & - \\
\hline Gentamicin & $1.78 \pm 0.12$ & $1.13 \pm 0.14 \boldsymbol{\Lambda}$ & - & $123.31 \pm 1.88$ & $78.89 \pm 4.08 \boldsymbol{\Delta}$ & - \\
\hline Cystone & $0.73 \pm 0.02$ & $0.08 \pm 0.04 \boldsymbol{\Lambda}$ & $1.05 \pm 0.09 \boldsymbol{\nabla}$ & $56.20 \pm 4.71$ & $11.78 \pm 2.52 \boldsymbol{\Lambda}$ & $67.11 \pm 6.60 \boldsymbol{\nabla}$ \\
\hline EP 200 mg/kg & $0.97 \pm 0.02$ & $0.31 \pm 0.007 \boldsymbol{\Delta}$ & $0.81 \pm 0.14 \boldsymbol{\nabla}$ & $113.26 \pm 1.81$ & $68.84 \pm 0.38 \boldsymbol{\Lambda}$ & $10.05 \pm 3.69 \boldsymbol{\nabla}$ \\
\hline EP $400 \mathrm{mg} / \mathrm{kg}$ & $0.84 \pm 0.01$ & $0.18 \pm 0.007 \boldsymbol{\Delta}$ & $0.94 \pm 0.13 \boldsymbol{\nabla}$ & $93.2 \pm 2.87$ & $48.78 \pm 5.06 \boldsymbol{\Lambda}$ & $30.11 \pm 0.98 \boldsymbol{\nabla}$ \\
\hline MN 200 mg/kg & $0.91 \pm 0.007$ & $0.26 \pm 0.01 \boldsymbol{\Delta}$ & $0.87 \pm 0.12 \boldsymbol{\nabla}$ & $99.99 \pm 2.02$ & $55.57 \pm 4.22 \boldsymbol{\Lambda}$ & $23.32 \pm 0.14 \boldsymbol{\nabla}$ \\
\hline MN 400 mg/kg & $0.79 \pm 0.02$ & $0.13 \pm 0.007 \boldsymbol{\Delta}$ & $0.99 \pm 0.15 \boldsymbol{\nabla}$ & $85.05 \pm 3.37$ & $40.63 \pm 1.18 \boldsymbol{\Lambda}$ & $38.26 \pm 5.26 \boldsymbol{\nabla}$ \\
\hline ME 200 mg/kg & $0.88 \pm 0.01$ & $0.22 \pm 0.007 \boldsymbol{\Delta}$ & $0.90 \pm 0.13 \boldsymbol{\nabla}$ & $94.68 \pm 4.6$ & $50.26 \pm 2.41 \boldsymbol{\Delta}$ & $28.63 \pm 6.49 \boldsymbol{\nabla}$ \\
\hline ME 400 mg/kg & $0.74 \pm 0.02$ & $0.09 \pm 0.04 \boldsymbol{\Lambda}$ & $1.04 \pm 0.09 \boldsymbol{\nabla}$ & $62.46 \pm 3.74$ & $18.04 \pm 5.93 \boldsymbol{\Lambda}$ & $60.85 \pm 1.85 \boldsymbol{\nabla}$ \\
\hline
\end{tabular}

EP E. pyriformis juice, MN M. nagi juice and ME M. esculenta juice. Inc Increase, Dec Decrease, $\mathbf{\Delta}$ Increase; $\mathbf{\nabla}$ Decrease

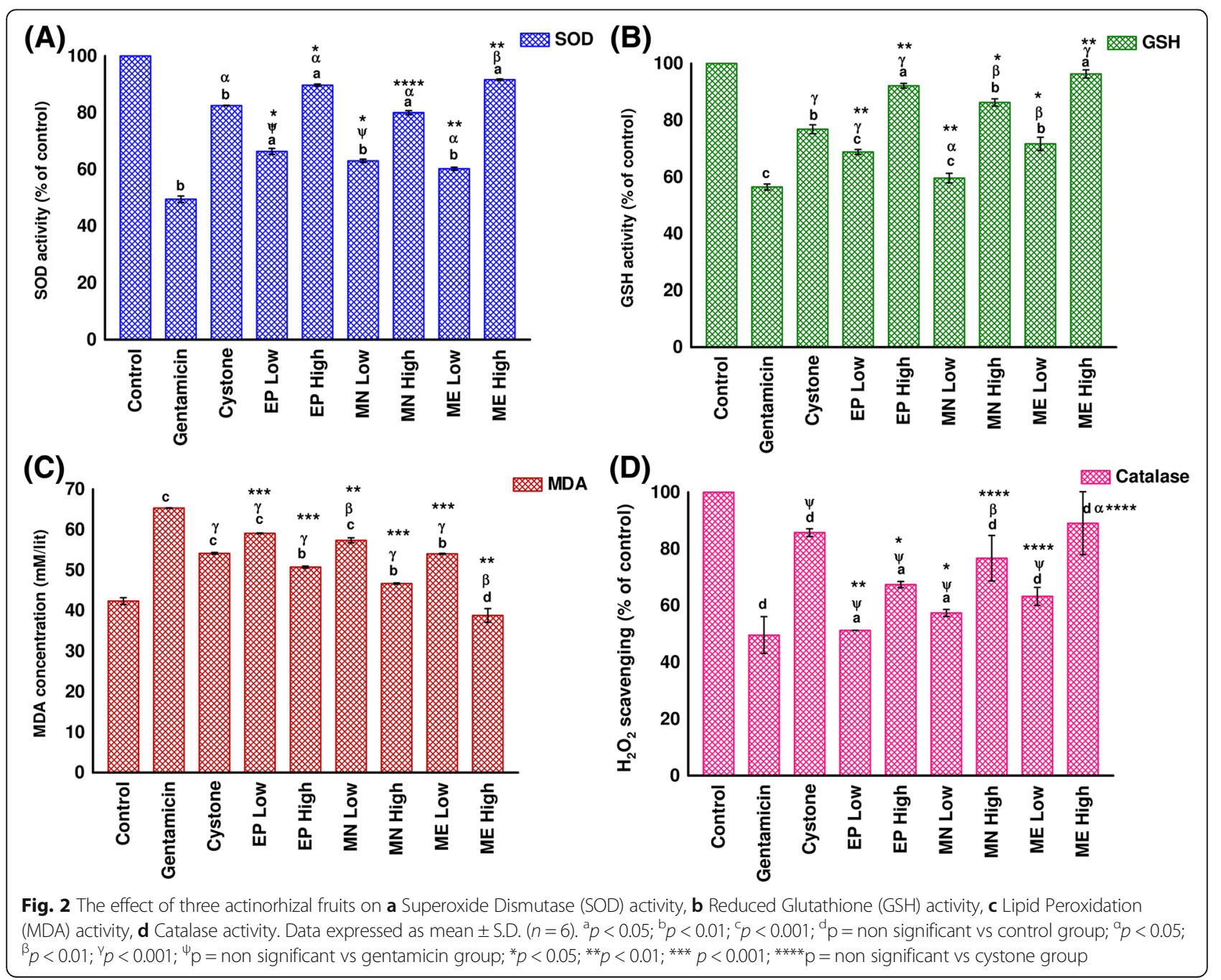




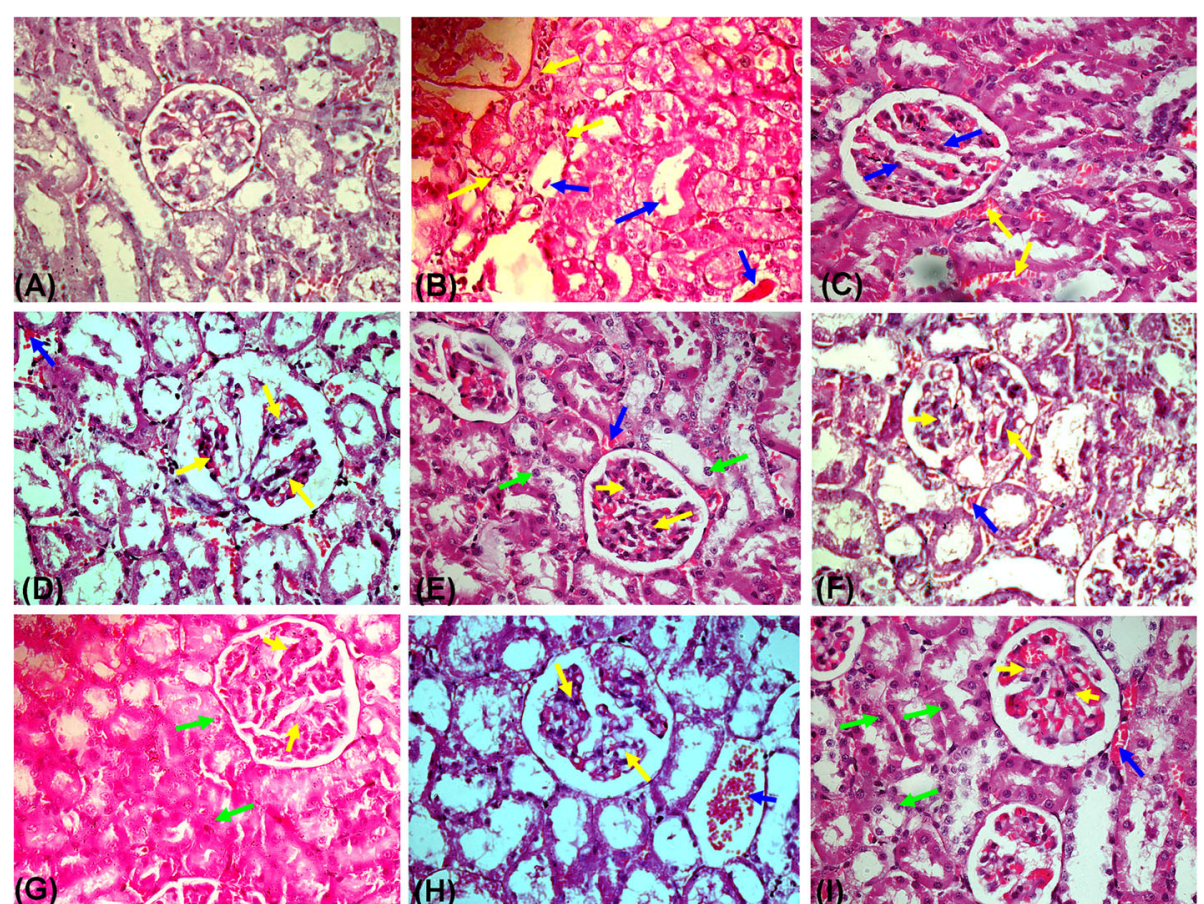

Fig. 3 Photomicrograph of control and treated rats kidney (A-l) a Control rats showing renal tubules and glomeruli are normal, b Gentamicin sulphate induced rat kidney showing severe intertubular hemorrhage (yellow arrows), degeneration, desquamation and necrosis in tubules (blue arrows), c Cystone exposed rats showing mild tubular epithelial changes; hyaline casts in tubules (blue arrows), low intertubular hemorrhage (yellow arrows), d E. pyriformis fruit juice (100 mg/kg BW) showing intertubular hemorrhage (yellow arrows), mild tubular epithelial changes; hyaline casts in tubules (blue arrow), e E. pyriformis fruit juice ( $400 \mathrm{mg} / \mathrm{kg} \mathrm{BW}$ ) showing low intertubular hemorrhage (yellow arrows), intact nucleus (green arrows), reduced hyaline casts in tubuler lumen (blue arrow), f M. nagi fruit juice (100 mg/kg BW) showing intertubular hemorrhage (yellow arrows), mild tubular epithelial changes; hyaline casts in tubules (blue arrow), g M. nagi fruit juice (400 mg/kg BW) showing low intertubular hemorrhage (yellow arrows), intact nucleus (green arrows), h M. esculenta fruit juice (100 mg/kg BW) showing intertubular hemorrhage (yellow arrows), mild tubular epithelial changes; hyaline casts in tubules (blue arrow), i M. esculenta fruit juice (400 mg/kg BW) showing low intertubular hemorrhage (yellow arrows), intact nucleus (green arrows), reduced hyaline casts in tubuler lumen (blue arrow)

urea level were found to be $0.65 \pm 0.02 \mathrm{mg} / \mathrm{dl}$ and $44.42 \pm 2.19 \mathrm{mg} / \mathrm{dl}$ respectively. Treating with gentamicin increased the creatinine and urea level by almost 2.74 and 2.78 fold.

\section{In-vivo antioxidant assays}

In this study four parameters were taken namely superoxide dismutase (SOD), Glutathione reductase (GSH), malonaldehyde (MDA) and catalase for in-vivo antioxidant assay. In all the cases AFJs showed promising results. In case of SOD, EPH and $\mathrm{MEH}$ attained the highest peak which was higher than Cystone (Fig. 2a). Similarly, in comparison to control, EPH, MNH and MEH showed better result than Cystone in case of GSH (Fig. 2b). Interestingly, MDA level was significantly decrease when treated with MEH which was even lower than the MDA level in control group (Fig. 2c). These indicate that consumption of $M$. esculenta juice not only reduced the kidney ailments but also made the rat healthier than the non treated rat. So far the catalase activity concerned, the MEH showed the catalase activity which was at par with Cystone (Fig. 2d).

\section{Histopathological examination}

Histopathological results demonstrate structural changes in renal tissue of different treatment groups. Histopathological examination of kidney of control and cystone group rat showed the normal renal tubules and glomeruli (Fig. 3a and c). Gentamicin considerably damages the kidney tissue physically leading to the deformation of glomerulus (Fig. 3b). It was found that treating with

Table 3 Molecular docking results showing compounds and their binding affinity

\begin{tabular}{ll}
\hline Compound name & Binding affinity (kcal/mol) \\
\hline 1,2-Longidione & -8.5 \\
2-Butenedioic acid (E)-, monomethyl ester & -5.1 \\
3-Hydroxydecanoic acid & -4.7 \\
5-Hydroxymethylfurfural & -5.1 \\
Adrenalone & -6.0 \\
Dodecanedioic acid & -4.7 \\
n-Hexadecanoic acid & -5.8 \\
Octadecanoic acid & -4.9 \\
\hline
\end{tabular}




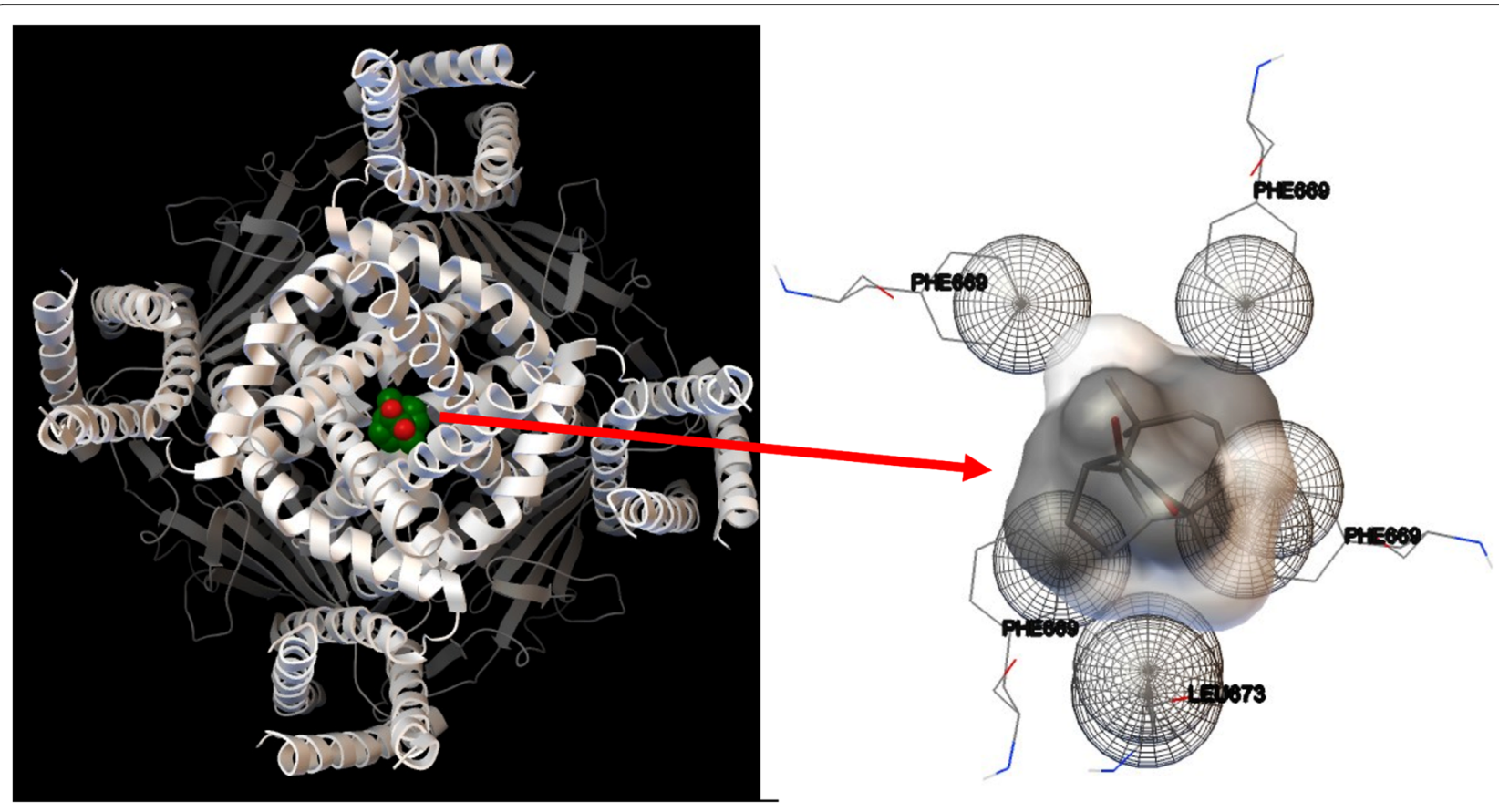

Fig. 4 In-silico docking representation of 1,2-Longidione with Polycystic Kidney Disease protein 2 (5T4D)

various AFJs significantly restored the glomerular structure to its normal form and all the studied fruit juices almost attributed equally in this regard (Fig. 3d-i).

\section{Molecular docking}

The three actinorhizal plants selected here provided loads of promising bioactive phytochemicals. We have reported the bioactivity of some of them previously [11]. However, we wanted to reinvestigate their potential from the point of view of renal health. We already have exciting result in these aspect considering results from the histopathological experiments and in-vivo enzymatic assays. The eight compounds mentioned in Table 3 are potent antioxidants. These compounds modulate and enhance enzymatic functions. As renal disfunctioning is much dependent on free radical and antioxidant imbalance, so the identified compounds in fruit juice serve in alleviating kidney problem [21, 22]. The activities of the compounds by molecular docking showed an overall good binding affinity (Table 3). The in-silico insight in this case showed that the compound 1,2-Longidione found in Myrica esculenta shows the best results with a binding affinity of $-8.5 \mathrm{kcal} / \mathrm{mol}$ in Autodock Vina (Fig. 4; Table 3), although, other promising compound like Adrenalone $(-6.0 \mathrm{kcal} / \mathrm{mol})$ and $\mathrm{n}$-Hexadecanoic acid $(-5.8 \mathrm{kcal} / \mathrm{mol})$ are not too far behind.

\section{Discussion}

Our study represents the first report to understand the efficacy of these juices to alleviate nephrotoxicity in rat model. It has been found that actinorhizal fruit juices (AFJs) are relatively better health supplements that the drug Cystone in managing kidney problems. Parenchyma tissues in kidney are very sensitive to natural or synthetic agents such as antimicrobials, chemotherapeutic agents and analgesics which begin to increase urea concentration only after parenchyma tissue injury [23]. When the rats were treated with Cystone and various fruit juices it has been found that in case of Cystone and high dose of $\mathrm{MEH}$, the creatinine level almost gone back to normal. All other AFJs also reduced the creatinine level. In case of urea test, Cystone (Group III) and MEH decrease the urea level considerably when compared with Gentamicin (Group II). These indicate that AFJs are effective in reducing the creatinine and urea level in diseased rats. Multiple mechanisms are involved in gentamicin nephrotoxicity such as generation of reactive oxygen species (ROS) and reactive nitrogen species (RNS) accumulation, which may lead to the glomerular congestion and acute tubular necrosis leading to diminished creatinine clearance and renal dysfunction [24, 25]. Overall scheme of action for AFJ derivatives were presented in Fig. 5. Catalase is an antioxidative enzyme that prevents the formation of highly reactive $\mathrm{OH}^{*}$ by scavenging $\mathrm{H}_{2} \mathrm{O}_{2}$, the key molecule of Fenton reaction. SOD (super oxide dismutase) and GSH (Glutathione reductase) alternatively catalyzes the dismutation of superoxide radicals into ordinary molecular oxygen or hydrogen peroxide. In this study, it is established that the diminished catalase, reduced glutathione and 


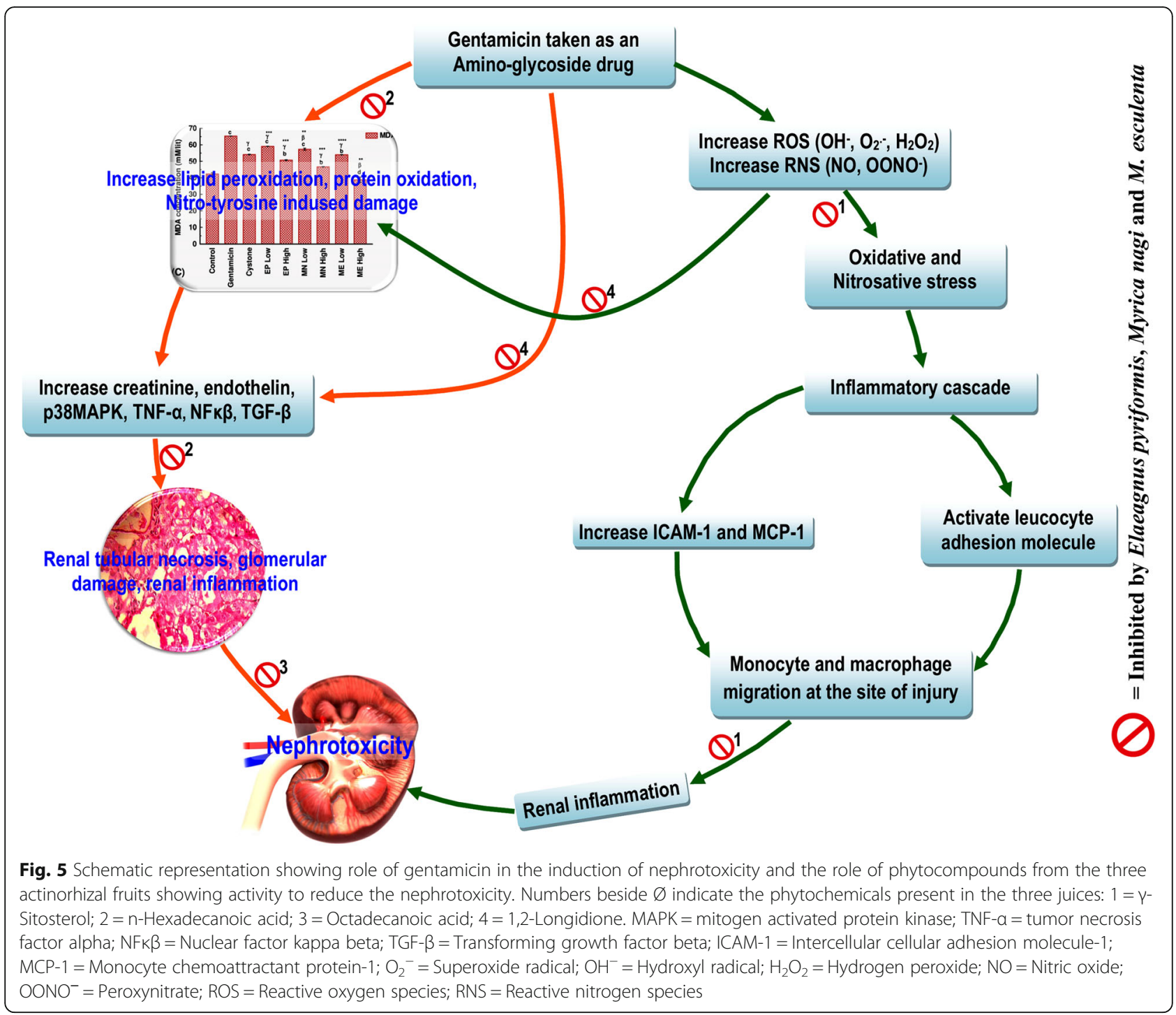

superoxide dismutase levels and elevated MDA levels were subsequently normalized by AFJs administration. The molecular docking results came out with new insights. In this study, the protein in consideration is Polycystic kidney disease protein 1-like 2 (PKD2) that is responsible for autosomal dominant polycystic kidney disease. This is one of the most important proteins responsible for multiple cyst formation in kidney [18]. The administration of these fruit juices showed thrilling result in the in-vivo as well as histopathological experiments. Later, in the light of in-silico experiments we may specify that compounds like 1, 2Longidione, adrenalone and n- Hexadecanoic acid might be the reason behind such exciting results. Nevertheless the query still remains whether 1, 2Longidione alone is capable of such effect or is it the combinatorial action as seen in most of the herbal formulations [26].

\section{Conclusion}

North-eastern part of India is rich in actinorhizal plants diversity and the local tribal people are familiar of the edible actinorhizal fruits and their therapeutic properties. The tribal groups are routinely consuming these fruits for various ailments including kidney problem. In the present study, we tried to analyze three actinorhizal fruit juices (AFJs) namely Elaeagnus pyriformis, Myrica nagi and M. esculenta for their efficacy in alleviating kidney disorders. We found that these fruit juices are quite efficient in keeping the kidney in healthy condition at least in albino rats. We also used a popular drug Cystone which is used worldwide for treating kidney disease as standard. Interestingly, we found some parameters the AFJs were performing better than Cystone. Among the three fruit juices $M$. esculenta particularly showed highly promising results by almost all means in the present study. We may therefore conclude that AFJs 
may be further exploited for formulating herbal medicine against kidney disorders. Besides, AFJs may be recommended as healthy dietary supplement for our day to day diet. So far the side effect of the juices is concerned; these fruit juices are used in North-east India since ages without any toxic effect.

\section{Acknowledgments}

This work was funded by Department of Biotechnology, Govt. of India. PK is thankful to Department of Biotechnology, Govt. of India for providing fellowship. AKC and SD acknowledge UGC, Govt. of India for providing fellowship.

\section{Authors' contributions}

PK conceived the idea. AS and TKC designed the experiments. PK, AKC, SD and AS carried out the research, collected the plant materials and analyzed data. PK, MB and SD carried the rat related experiments. AKC contributed in in-silico analysis. All authors wrote the MS and approved it.

\section{Funding}

The present study was not supported by any external funding agencies.

\section{Availability of data and materials}

The supporting materials can be obtained upon request via email to the corresponding author.

\section{Ethics approval and consent to participate}

All the experiments were approved by the ethical committee, University of North Bengal (No. 840/ac/04/CPCSEA) and conducted in accordance with the legislation for the protection of animals used for scientific purposes. The study does not include human subjects.

\section{Consent for publication}

Human volunteers are not included in this study, so, their consent is not applicable. All the authors have given their consent for this publication.

\section{Competing interests}

The authors declare that they have no competing interests.

\section{Author details}

'Department of Botany, University of North Bengal, Siliguri, West Bengal 734013, India. ²Department of Zoology, University of North Bengal, Siliguri, West Bengal 734013, India. ${ }^{3}$ Department of Tea Science, University of North Bengal, Siliguri 734013, India. ${ }^{4}$ Department of Zoology, Bodoland University, Kokrajhar, Assam 783370, India.

Received: 19 November 2018 Accepted: 29 May 2019

Published online: 14 June 2019

\section{References}

1. Young B, Heath JW, Stevens A, Lowe JS, Deakin PJ. Wheater's Functional Histology. 5th ed: Churchill Livingstone. Oxford: Elsevier Limited; 2006.

2. Kumar P, Clark M, editors. Clinical Medicine: W.B. Saunders. UK: Harcourt Publishers Limited; 2001.

3. Alchi B, Nishi S, Kondo D, Kaneko Y, Matsuki A, Imai N, Ueno M, Iguchi S, Sakatsume M, Narita I, Yamamoto T. Osteopontin expression in acute renal allograft rejection. Kidney Int. 2005;67:886-96.

4. Ferguson MA, Vaidya $\mathrm{V}$, Bonventre JV. Biomarkers of nephrotoxic AKI. Toxicol. 2008;245:182-93.

5. Abdel-Raheem IT, Abdel Ghany AA, Mohamed GA. Protective effect of quercetin against gentamicin-induced nephrotoxicity in rats. Biol Pharm Bull. 2009;32:61-7.

6. Elfarra AA, Duescher RJ, Sausen PJ, Ohara TM, Cooley AJ. Methimazole protection of rats against gentamicin-induced nephrotoxicity. Can J Physiol Pharmacol. 1994;72:1238-44.

7. Hayelom K, Mekbeb A, Eyasu M, Wondwossen E, UMethanolic K. Effect of Clerodendrum myricoides root extract on blood, liver and kidney tissues of mice. Afr Health Sci. 2012;12:489-97.

8. Ribeiro A, Berry AM, Pawlowski K, Santos P. Actinorhizal plants. Funct Plant Biol. 2011;38:5-7.
9. Sharma SD, Kumar K. Temperate fruit diversity status and prospects. In: Kishore DK, Sharma SK, Pramanick KK, editors. Temperate Horticulture: Current Scenario. New Delhi: New India Publishing Agency; 2006. p. 27-34.

10. Goyal AK, Mishra T, Bhattacharya M, Kar P, Sen A. Evaluation of phytochemical constituents and antioxidant activity of selected actinorhizal fruits growing in the forests of Northeast India. J Biosci. 2013;38:797-803.

11. Kar P, Dey P, Misra AK, Chaudhuri TK, Sen A. Phytometabolomic fingerprinting of selected actinorhizal fruits popularly consumed in NorthEast India. Symbiosis. 2016;70:159-68.

12. OECDiLibrary. OECD Guidelines for the testing of chemicals, Section 4: Health effects; 2002. https://doi.org/10.1787/9789264071001-en.

13. Dutta S, Chakraborty AK, Dey P, Kar P, Guha P, Sen S, Kumar A, Sen A, Chaudhuri TK. Amelioration of $\mathrm{CCl}_{4}$ induced liver injury in swiss albino mice by antioxidant rich leaf extract of Croton bonplandianus Baill. PLoS One. 2018;13:e0196411.

14. Misera HP, Fridovich I. The role of superoxide anion in the auto-oxidation of epinephrine and a simple assay for SOD. J Biol Chem. 1972;247:3170-5.

15. Ellman GL, Courtney KD, Andres V, Featherstone RM. A new and rapid colorimetric determination of acetylcholinesterase activity. Biochem Pharmacol. 1961:7:88-95.

16. Luck H, editor. Methods of enzymatic analysis. New York: Academic Press; 1963.

17. Dey P, Dutta S, Biswas-Raha A, Sarkar MP, Chaudhuri TK. Haloalkane induced hepatic insult in murine model: amelioration by oleander through antioxidant and anti-inflammatory activities, an in-vitro and in-vivo study. BMC Complement Altern Med. 2016;16:280-95.

18. Yoder BK, Hou X, Guay-Woodford LM. The polycystic kidney disease proteins, polycystin-1, polycystin-2, polaris, and cystin, are co-localized in renal cilia. J Am Soc Nephrol. 2002;13:2508-16.

19. Levine E, Grantham JJ. Calcified renal stones and cyst calcifications in autosomal dominant polycystic kidney disease: clinical and $C T$ study in 84 patients. Am J Roentgenol. 1992;159:77-81.

20. Trott O, Olson AJ. Auto dock Vina: improving the speed and accuracy of docking with a new scoring function, efficient optimization, and multithreading. J Comput Chem. 2010;31:455-61.

21. Zhao L, Chen J, Su J, Li L, Hu SLB, Chen T. In vitro antioxidant and antiproliferative activities of 5-hydroxymethylfurfural. J Agric Food Chem. 2013; 61:10604-11.

22. Aparna V, Dileep KV, Mandal PK, Karthe P, Sadasivan C, Haridas M. Antiinflammatory property of n-Hexadecanoic acid: structural evidence and kinetic assessment. Chem Biol Drug Des. 2012;80:434-9.

23. Gilbert DN, Wood CA, Kohlhepp SJ, Kohnen PW, Houghton DC, Finkbeiner HC, Lindsley J, Bennett WM. Polyaspartic acid prevents experimental aminoglycoside nephrotoxicity. J Infect Dis. 1989:159:945-53.

24. Mingeot-Leclercq MP, Tulkens PM. Aminoglycosides: nephrotoxicity. Antimicrob Agents Chemother. 1999:43:1003-12.

25. Hur E, Garip A, Camyar A, Ilgun S, Ozisik M, Tuna S, Olukman M, Narli Ozdemir Z, Yildirim-Sozmen E, Sen S, Akcicek F. The effects of vitamin D on gentamicin-induced acute kidney injury in experimental rat model. Int J Endocrinol. 2013:2013:1-7.

26. Chalannavar RK, Narayanaswamy VK, Baijnath H, Odhav B. Chemical constituents of the essential oil from leaves of Psidium cattleianum var. cattleianum. J Med Plants Res. 2013:7·783-9.

\section{Publisher's Note}

Springer Nature remains neutral with regard to jurisdictional claims in published maps and institutional affiliations. 